\title{
Design of Laboratory Bench for Fluorescent Lamp Power Factor
}

\author{
Hanpin $\mathrm{Luo}^{1, \mathrm{a}}$, Facai Qiü,a, Jianbo Cao ${ }^{1, \mathrm{~b}_{*}}$, Qifen $\mathrm{Gu}^{1, \mathrm{c}}$, Zhongyao $\mathrm{Wu}^{1, \mathrm{c}}$, \\ Quanhui Sun ${ }^{3, \mathrm{c}}$ and Tianfeng Zhao ${ }^{1, \mathrm{c}}$ \\ ${ }^{1}$ College of Engineering, Zhejiang Normal University, Jinhua, Zhejiang 321004, P.R. China \\ ${ }^{2}$ Shandong Yellow River Materials Storage Center, Jinan, Shandong 250032, P.R. China \\ ${ }^{3}$ Jinan Yellow River Engineering Bureau, Jinan, Shandong 250013, P.R. China

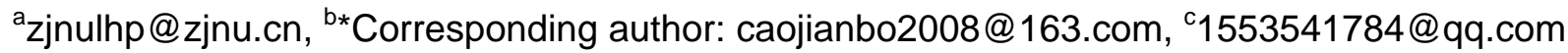

Keywords: Fluorescent lamp, Power factor, Mechanical design, Laboratory bench

\begin{abstract}
The circuit experiment and the power factor improvement experiment of fluorescent lamp are the two traditional experiment items in electrotechnics. They are quite important for students to master the basic theory and acquire the experiment skills. A new kind of convenient fluorescent lamp power factor laboratory bench was designed based on the study of the basic structure and the working principle of the fluorescent lamp circuit. Its 3D modeling was also designed by Solidworks software. The laboratory bench is simple and safe in operation and help teaching. It has good value of application.
\end{abstract}

\section{Introduction}

With the rapid development of economy, the social requirement of electricity is increasing. A huge drain on energy and low resource utilization are the attendant prominent problems. Handling the question of the distribution of active power and reactive power in the system, that is to improve the power factor in the circuit, is the key way to improve power energy utilization rate. Improving the power factor in the circuit is a vital aspect and way of saving energy because the fluorescent circuit could send out a bundle of bright, softness light and also it is practical and economical and wildly used in any occasions [1]. At the same time, it is necessary to develop new-style laboratory bench for fluorescent lamp power factor so as to help students understand the alternating current circuit and the power factor through the study of college electrotechnics education and experimentation status. So in this paper the author designed a new kind of convenient fluorescent lamp power factor laboratory bench to improve the present situation of experiment teaching and teaching level, and benefit the professional engineering students.

\section{Research on the Work Principle and Power Factor of Fluorescent Lamp}

The fluorescent lamp circuit is made up of lamp tube, ballast, starter, etc. Each part of the structure is shown in Fig. 1.

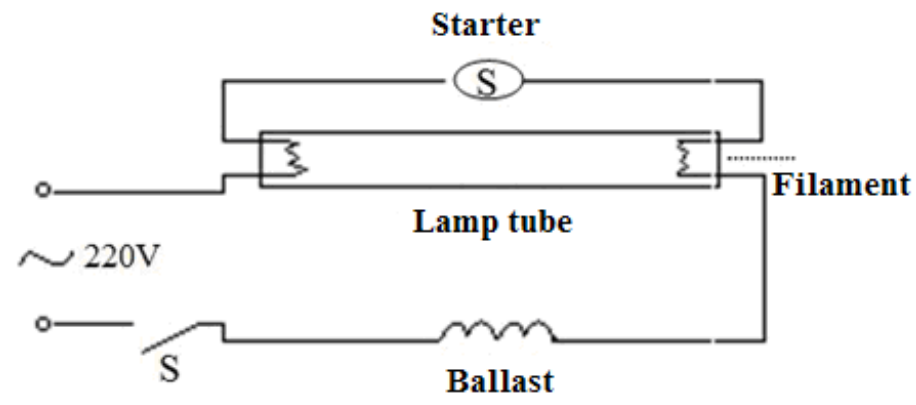

Fig. 1 Connection diagram of the fluorescent lamp circuit 
Calculating the average power of AC should take the phase difference of voltage and current, i.e. $\varphi$, into consideration.

$P=U I \cos \varphi$

The $\cos \varphi$ mentioned the formula is the power factor of the circuit. The phase difference of voltage and current or the power factor of the circuit is dependent on the circuit (load) parameters.

In AC circuits, there are two kinds of electric powers for power supplying the load: one is active power $\mathrm{P}$, the other is reactive power $\mathrm{Q}$. Active power is to maintain the electric power for the normal operation of the electrical equipments, and that is to transform the electric energy into other forms of energy (mechanical energy, light and heat).Reactive power seems abstract, and it is used for the transformation between the electric field and the magnetic field of the circuit, and it is used for the establishment and maintenance of magnetic field in the electrical equipments [2]. Apparent Power $S=U I=\sqrt{P^{2}+Q^{2}}$. In electrotechnics, power factor could also indicate the utilization rate of the alternating current (AC). Power factor defined as the ratio of active-power $p$ and apparent power $\mathrm{S}$, that is $\cos \varphi=P / S$ [3-5].

Fluorescent lamp is inductive load and the commonly used methods for improving the power factor is to the perceptual load, the common way to improve power factor is to parallelly connect the inductive load and electrostatic capacitance. Its circuit diagram and phasor diagram as shown in Fig. 2 [6-7].

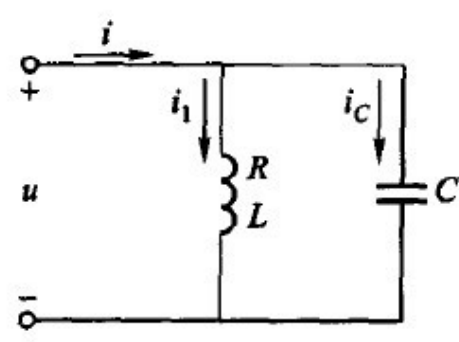

(a) Circuit diagram

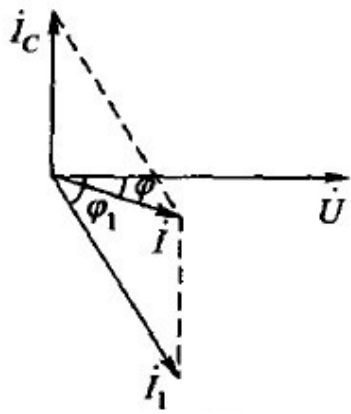

(b) Phasor diagram

Fig. 2 Capacitor and inductive load in parallel connection

The electric current and the power factor of inductive load would not change after parallelly connecting the capacitor because the applied voltage and load parameter hasn't been changed while the phase difference $\varphi$ of the voltage $\mathrm{u}$ and electric current has became declined, that is $\cos \varphi$ rising. Here the factor of improving power mentioned is the factor of improving the power supply or the power grid, rather than any inductive load power factor.

In theory, it is sure to find a compensation capacitor to make the $\cos \varphi$ of the circuit which is compensated by the capacitor 1 . But it's hard to get " 1 " due to the restriction of experimental equipment and the limit of electric network.

The experiment needs to measure the power $\mathrm{P}$ of main current, power factor $\cos \varphi$, voltage $U$, current $I$, the power $p_{1}$ of branch current, power factor $\cos \varphi_{1}$ and current $I_{R}$. According to the principle—series circuit connection ahead of parallel circuit connection, as shown in Fig. 3.

\section{Design of Laboratory Bench for Fluorescent Lamp Power Factor}

Fluorescent lamp power factor laboratory bench is a kind of experimental equipment for the electrotechnics teaching. First and foremost, the experiment requests for the laboratory bench to offer fluorescent lamp with $220 \mathrm{~V}-50 \mathrm{HZ}$ AC working voltage and get through fluorescent lamp circuit. Second the laboratory bench should be installed the corresponding measuring instrument to measure all kinds of electronic data of fluorescent lamp work circuit, such as voltage, current, power, power factor, etc. Furthermore, he laboratory bench should have the function of installing and uninstalling capacitor easily so as to carry out the power factor improvement experiment. In the paper the author 
designed the 3D modeling of the fluorescent lamp power factor laboratory bench as shown in Fig. 4 Fig. 6. In this solution, the tank cover and the tank bottom were all made by aluminium alloy.The front panel of the tank was cut out the electronic measurement instrument, the power switch and the capacitor and the interface of multimeter by the laser cutter, and the electronic measurement instrument and plug were fixed on the bottom, and a drawer was designed in the right part of the tank bottom so as to store the capacitor and multimeter.

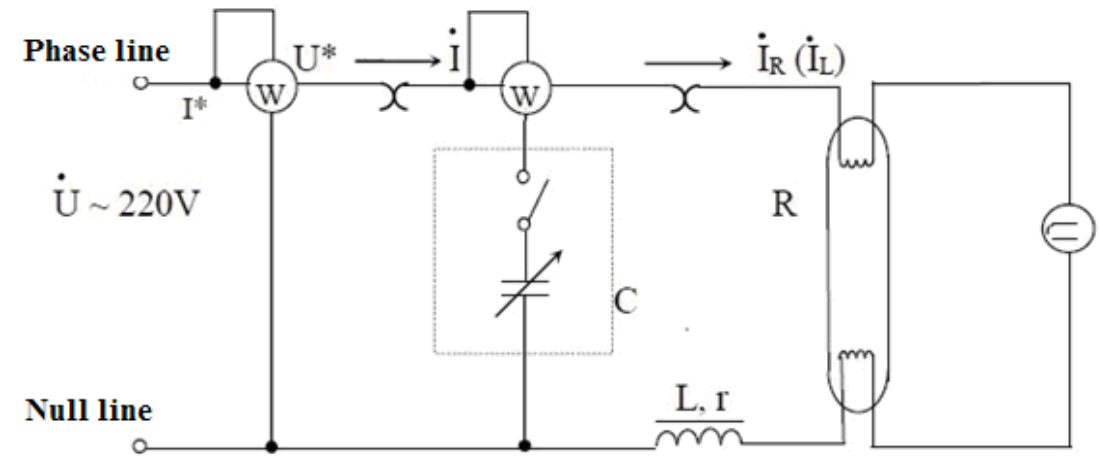

Fig. 3 Experimental diagram of fluorescent lamp parallel connection with capacitor

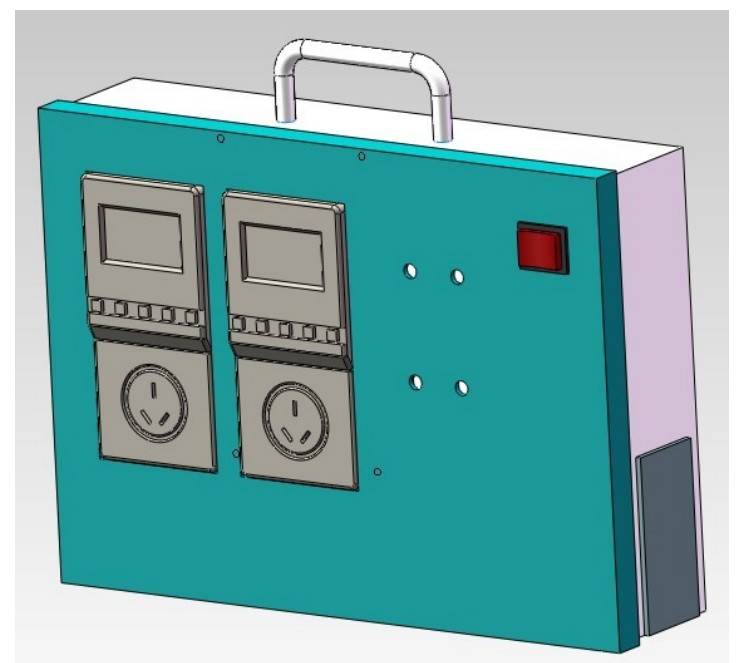

Fig. 4 First 3D modeling of laboratory bench

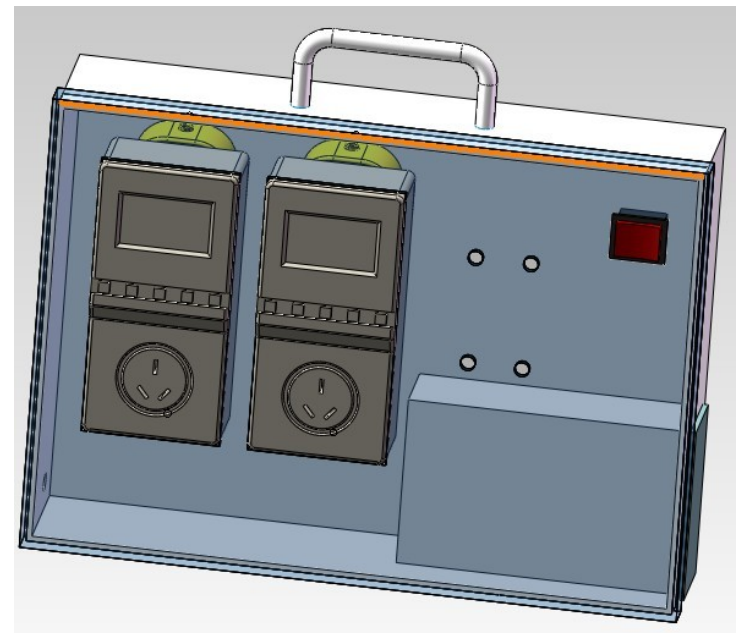

Fig. 5 Second 3D modeling of laboratory bench 


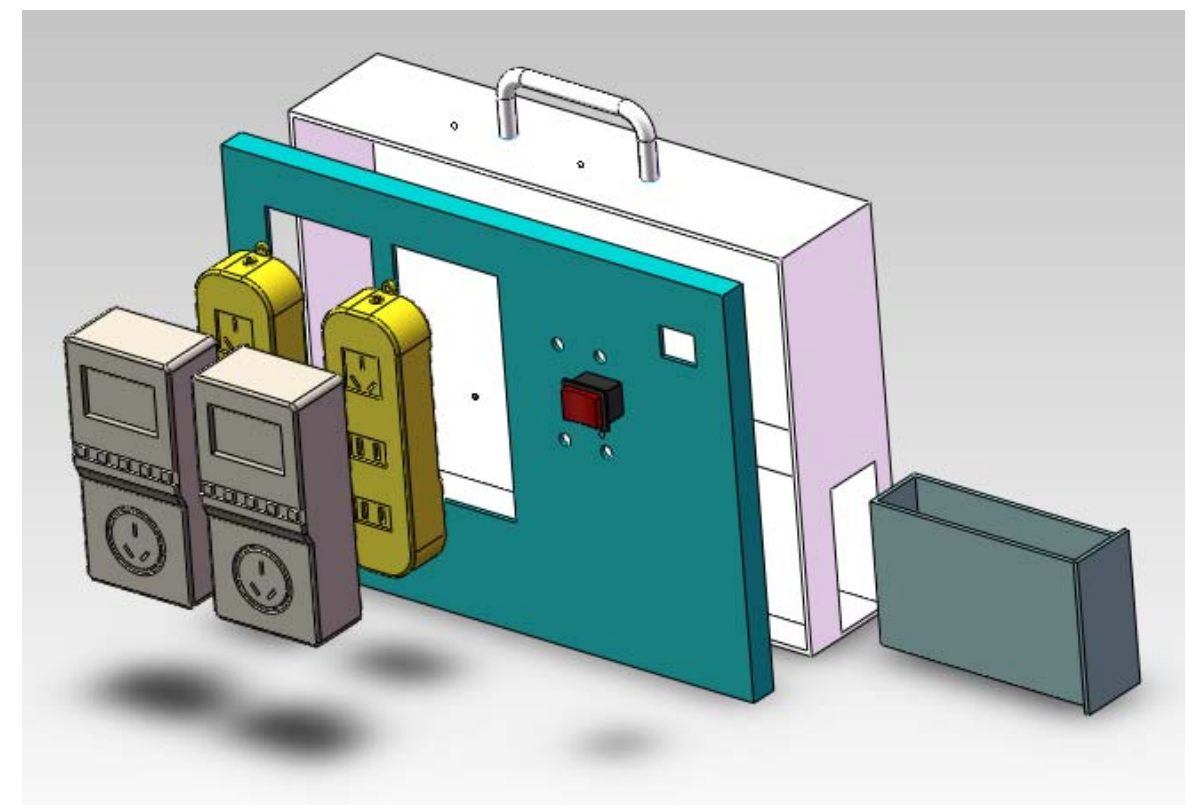

Fig. 6 Third 3D modeling of laboratory bench

\section{Conclusion}

In this paper the content was closely combined with the study of college electrotechnics contents of courses and make up for the deficiency of the experimental equipments in the fluorescent lamp and power factor. The experimental teaching could make the knowledge more impressive and clear to be acquired by students. In this paper the design of laboratory bench for fluorescent lamp power factor used parallel capacitor circuit. The method of improving the power factor of inductive circuit could not only make the circuit simplified, safety-improved, but also help students understand this part of the teaching content in the experiment. The appearance of the designed laboratory bench for fluorescent lamp power factor is simple in design, cheap in cost, and has good value of popularization and application.

\section{Acknowledgement}

The work was Supported by the Key Project of Chinese Ministry of Education (No. 211071), the Science and Technology Project of Jinhua City (No. 2010-1-101), and the Experimental Technology Development Project of Zhejiang Normal University (No. sj201216).

\section{References}

[1] J. Wang: Experimental Science and Technology, Vol. 5 (2007) No. 1, pp. 23-25.

[2] J.X. Lu: Journal of Beijing Industrial Vocational and Technical College, Vol. 3 (2004) No. 4, pp. 91-94.

[3] F.V. Topalis, I.F. Gonos and G.A. Vokas: Measurement, Vol. 30 (2001), pp. 257-267.

[4] C.H. Xu, H.T. Zhuang, Y.J. Zhong and W.D. Zhu: Journal of Zhejiang Normal University (Natural Sciences), Vol. 34 (2011) No. 4, pp. 429-435.

[5] J.C.W. Lam and P.K Jain: IEEE Transactions on Power Electronics, Vol. 25 (2010) No. 8, pp. 2045-2058.

[6] C.B. Nascimento and A.J. Perin: IEEE Transactions on Power Electronics, Vol. 55 (2008) No. 2, pp. 2045-2058.

[7] Y.C. Chuang and H.L. Cheng: IEEE Transactions on Industry Applications, Vol. 46 (2007) No. 6, pp. 1434-1440. 